\title{
Effet du confinement sur la cavitation de tourbillon marginal
}

\author{
O. Boulon, J.P. Franc, J.M. Michel
}

Laboratoire des Ecoulements Géophysiques et Industriels

Institut de Mécanique de Grenoble - UJF - CNRS - INPG

\section{I $\square$ CADRE DE L'ÉTUDE ET MOYENS EXPÉRIMENTAUX}

Le problème de l'enroulement tourbillonnaire qui prend naissance à l'extrémité d'une aile d'envergure limitée, telle qu'une pale d'hélice, a fait l'objet de nombreuses études, tant expérimentales que théoriques. En France, des moyens importants ont été mis en ouvre par la Direction de la Recherche et de la Technologie de 1991 à 1995, pour développer une action de recherche concertée entre plusieurs centres, sur ce thème [1].

Pour sa part, le LEGI a été chargé, entre autres, de l'étude de l'effet de confinement. L'objectif est d'analyser comment l'enroulement tourbillonnaire est modifié par la présence d'une paroi, comme c'est le cas pour les hélices carénées. Nous nous sommes attachés à analyser l'influence du jeu d'entrefer sur les conditions d'apparition de la cavitation dans le tourbillon marginal. Les modifications entraînées par le confinement sur la structure subcavitante du tourbillon ont également fait l'objet d'une étude détaillée, sur la base de mesures de vitesses par anémométrie laser.
L'étude présentée ici est essentiellement expérimentale. Elle a été menée en parallèle avec une étude théorique qui fait l'objet d'une deuxième présentation dans le cadre de ces journées [2]. Les essais ont été réalisés dans le tunnel hydrodynamique du Laboratoire des Ecoulements Géophysiques et Industriels de l'Institut de Mécanique de Grenoble. La configuration d'essais est schématisée sur la figure 1.

Elle comprend une aile, de forme en plan elliptique, dont la section droite est un profil de type Naca 16-020, de $20 \%$ d'épaisseur relative. La corde à l'emplanture, c, est de $120 \mathrm{~mm}$, l'envergure, b, est de $180 \mathrm{~mm}$, et l'allongement, $8 \mathrm{~b} / \pi \mathrm{c}$, vaut 3,8 . Le profil est implanté verticalement dans la veine d'essais, en charge, de hauteur $280 \mathrm{~mm}$ et de largeur $175 \mathrm{~mm}$. Le confinement est réalisé à l'aide d'une plaque plane horizontale dont la distance à l'extrémité de l'aile, l'entrefer e, est continuement réglable de 0 à $80 \mathrm{~mm}$.

Nous avons testé plusieurs plaques, dont les longueurs, en amont de l'extrémité de l'aile, étaient différentes. L'objectif initial était d'analyser l'interaction entre la couche limite de plaque et le tourbillon marginal, les diverses plaques permettant de

\section{Schéma de principe du montage} expérimental.

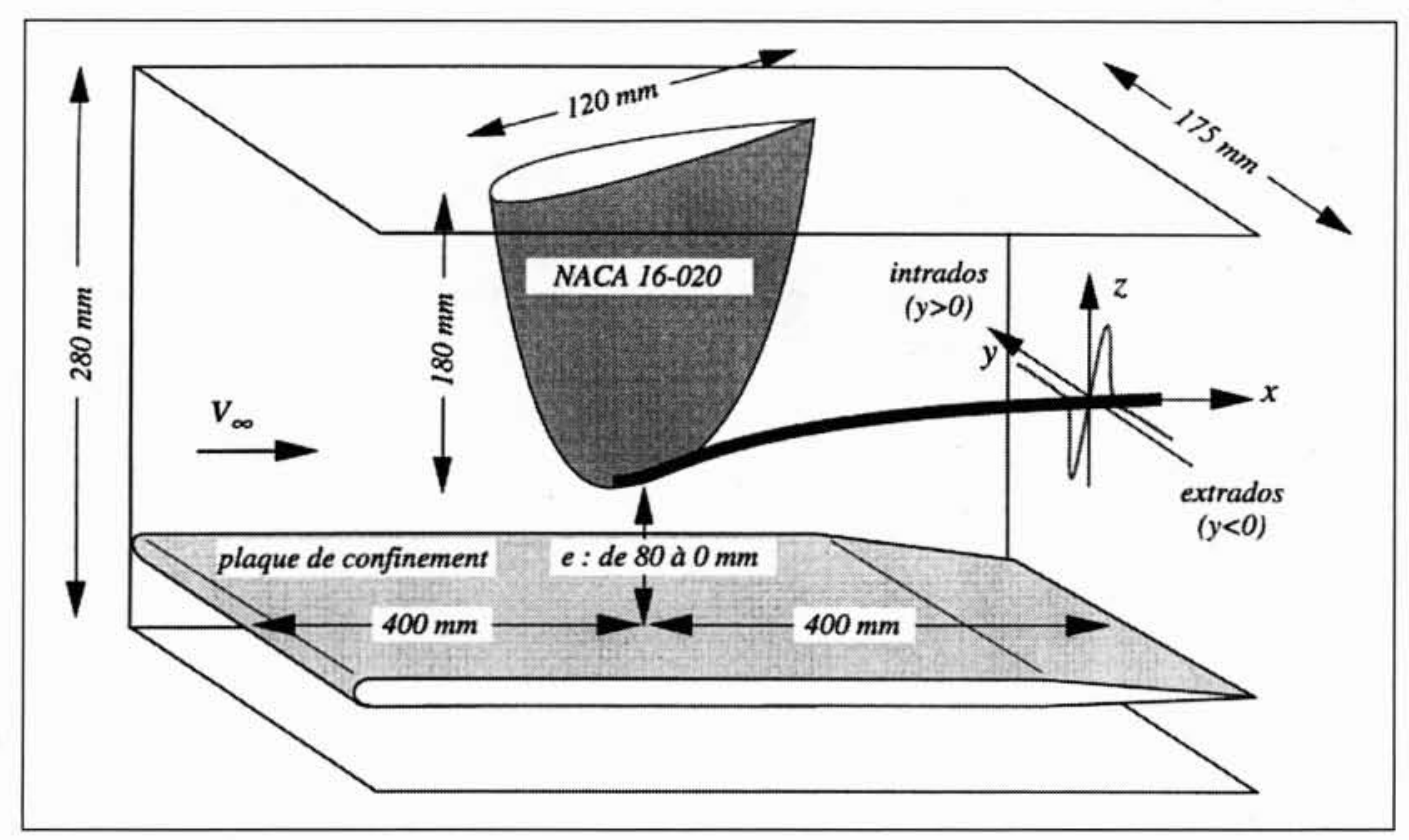


générer diverses qualités de couche limite, laminaire ou turbulente, d'épaisseurs différentes. Les résultats correspondants ne sont pas présentés ici, car les effets de type visqueux attendus se sont avérés très secondaires devant l'effet d'origine potentiel. Cela s'explique par le fait que, même pour la plaque la plus longue considérée dans cette étude, l'épaisseur de déplacement de la couche limite n'est que de quelques dixièmes de millimètre. La contraction du sillage fait que le tourbillon échappe très vite à la zone de déficit de vitesse de paroi et qu'il n'est donc quasiment pas affecté par la couche limite.

La vitesse d'écoulement peut varier entre 3 et $12 \mathrm{~m} / \mathrm{s}$, ce qui correspond à un nombre de Reynolds, basé sur la corde à l'emplanture, compris entre 0,36 et 1,4 . 10 $0^{6}$. La plupart des essais présentés ici ont été menés à un nombre de Reynolds, de l'ordre de 900000 .

Les mesures de vitesse ont été réalisées grâce à un anémomètre laser DANTEC de type FlowLite, opérant en rétrodiffusion. L'optique d'émission et de réception est équipée d'un élargisseur de faisceaux. Il permet de réduire la taille de la zone d'interférences à $0,7 \mathrm{~mm}$ x 0,078 $\mathrm{mm}$, et ainsi, de l'adapter aux mesures de vitesse tangentielle dans le cœur visqueux, de très petite taille. Le volume de mesure effectif se trouve encore réduit par le jeu des traitements appliqués au signal doppler. L'écoulement est ensemencé en particules d'iriodine, afin d'améliorer la qualité du signal ainsi que les taux d'acquisition et de validation.

\section{II — VISUALISATIONS}

Une campagne de visualisation a été menée dans diverses conditions de vitesse, de pression et d'incidence. Dans chaque cas, l'entrefer était progressivement réduit de quelques dizaines de millimètres à quelques dixièmes de millimètre seulement. La figure 2 présente une série typique de visualisations, pour un confinement de plus en plus important.

Les conditions de départ sont non cavitantes et correspondent à un entrefer de $50 \mathrm{~mm}$. Pour cette valeur, un léger effet de confinement se fait déjà sentir, comme l'a montré l'approche numérique [3], par comparaison au cas du milieu infini ; il reste cependant sans commune mesure avec celui qui est mis en évidence ici, pour des entrefers bien inférieurs.

Lorsque l'entrefer diminue, toutes choses égales par ailleurs, la figure 2 montre que, pour la valeur du paramètre de cavitation considérée ici ( 2,6 en l'occurence), le tourbillon marginal ne cavite pas tant que l'entrefer reste supérieur à $20 \mathrm{~mm}$.

Pour un entrefer de l'ordre de $20 \mathrm{~mm}$, un tube de vapeur apparaît assez soudainement, de façon stable, mais non attachée à l'extrémité de l'aile, comme le montre la deuxième photographie. La distance, vers l'aval, entre le bout d'aile et l'extrémité du filament est de l'ordre de $15 \mathrm{~mm}$, ce qui correspond à une valeur adimensionnelle $\mathrm{x} / \mathrm{c}$ de l'ordre de 0,12 . On peut se demander si ce point particulier ne correspondrait pas au point de pression minimale, le long de la trajectoire du tourbillon. Car, si la pression était inférieure en amont de ce point, on comprendrait difficilement qu'il n'y ait pas vaporisation dans cette zone. Dans l'état actuel des recherches, il ne s'agit, cependant, que d'une conjecture.

Lorsque l'entrefer continue de diminuer, le tube de vapeur s'allonge en direction de l'extrémité de l'aile ; pour un entrefer de $13 \mathrm{~mm}$, il atteint le bout de l'aile. Les conditions critiques de cavitation qui ont été considérées dans cette étude sont précisément les conditions d'accrochage du tube de vapeur à l'extrémité du profil. Elles correspondent à un état de cavitation sensi- blement plus développé que la cavitation naissante. Mais, de façon générale, les conditions d'accrochage du tourbillon sont très reproductibles, contrairement au critère basé sur l'apparition de la "première bulle" qui est très sensible, en particulier, à la teneur de l'eau en germes. Les conditions d'accrochage du tube de vapeur à l'extrémité de l'aile correspondent à l'instant où la vapeur, initialement présente au cœur du tourbillon dans la masse du fluide, atteint le bulbe de décollement laminaire attaché au bord d'attaque de l'aile. Les visualisations montrent qu'il y a continuité de structure entre le bulbe de décollement de bord d'attaque, qui est une structure attachée, et le tourbillon marginal, qui est une structure libre.

Pour des valeurs inférieures du jeu d'entrefer, la cavitation de tourbillon marginal gagne progressivement le bord d'attaque de l'aile pour donner naissance à une petite poche attachée. Cette poche, initialement limitée à l'extrémité même de l'aile, s'étend progressivement vers l'emplanture lorsque le confinement augmente. On distingue très nettement deux zones différentes sur l'interface (voir par exemple la photographie correspondant à $e=6 \mathrm{~mm}$ ). La partic la plus proche du bout de l'aile est fortement irisée, ce qui témoigne d'un régime d'écoulement turbulent audessus de la poche. Au contraire, la partie la plus éloignée du bout de l'aile exhibe une interface lisse, caractéristique d'un régime d'écoulement laminaire. Ces observations prouvent que la transition à la turbulence affecte, en premier, l'extrémité de pale.

La cavité de bord d'attaque, qui s'était progressivement développée en augmentant le confinement, amorce une décroissance en taille, depuis l'emplanture, au fur et à mesure que l'entrefer diminue, au-dessous de $6 \mathrm{~mm}$ environ. Le tourbillon cavitant se trouve fortement perturbé et n'est quasiment plus visible pour un entrefer de $2 \mathrm{~mm}$.

A très fort confinement, pour un entrefer de $0,5 \mathrm{~mm}$ seulement, la cavitation a presque totalement disparu, qu'il s'agisse de la cavité de bord d'attaque ou de la cavitation de tourbillon marginal. La cavitation se manifeste plutôt par des petites structures très éphémères qui sont caractéristiques des structures tourbillonnaires tridimensionnelles généralement observées dans les zones décollées des sillages épais. Nous reviendrons sur ce point au paragraphe $\mathrm{V}$.

\section{III $\square$ CONDITIONS CRITIQUES DE CAVITATION}

Nous présentons, dans ce paragraphe, les résultats relatifs à l'effet du confinement sur les conditions critiques de cavitation. Le critère retenu est la configuration limite correspondant à l'attachement du tube de vapeur à l'extrémité de l'aile. Comme expliqué au paragraphe précédent, la naissance de la cavitation dans le tourbillon marginal, caractérisée par l'explosion de la première bulle, se produit préalablement à l'obtention d'un tube de vapeur continu et attaché. Ce dernier critère, très reproductible, donne cependant une bonne image des conditions de cavitation naissante.

Nous avons porté, sur la figure 3 , les variations du paramètre de cavitation critique, en fonction de l'angle d'incidence, pour plusieurs jeux d'entrefer. Ces courbes font apparaître une très forte influence du confinement sur les conditions critiques de cavitation : à incidence fixée, la cavitation est initiée, dans le tourbillon, pour des nombres de cavitation beaucoup plus grands, en milieu confiné. A 8 degrés par exemple, l'apparition du tube de vapeur se produit en milieu non confiné $(\mathrm{e}=60 \mathrm{~mm})$ pour un nombre de cavitation proche de 1 , alors qu'en milieu confiné $(\mathrm{e}=2 \mathrm{~mm})$, elle se produit pour un nombre de cavitation égal à 4. 

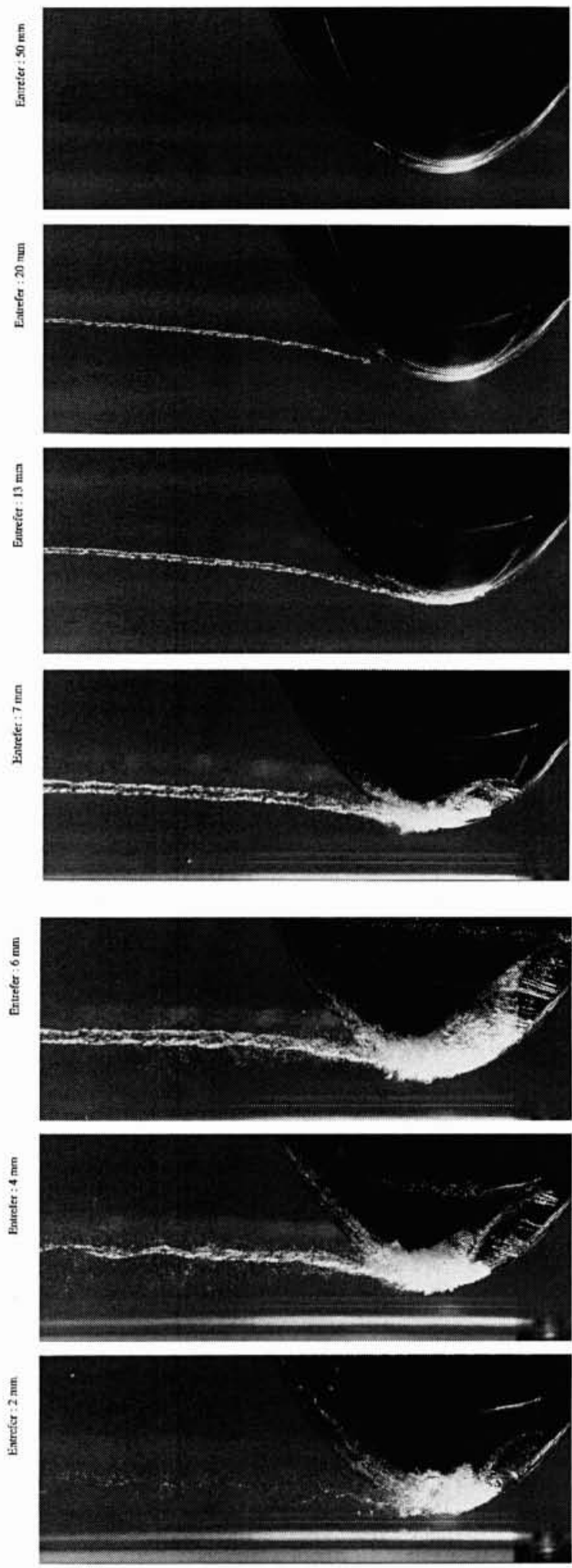

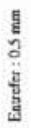

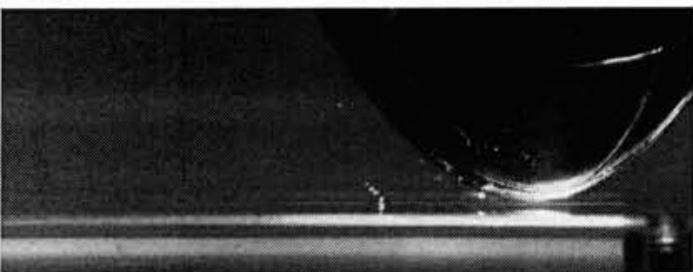

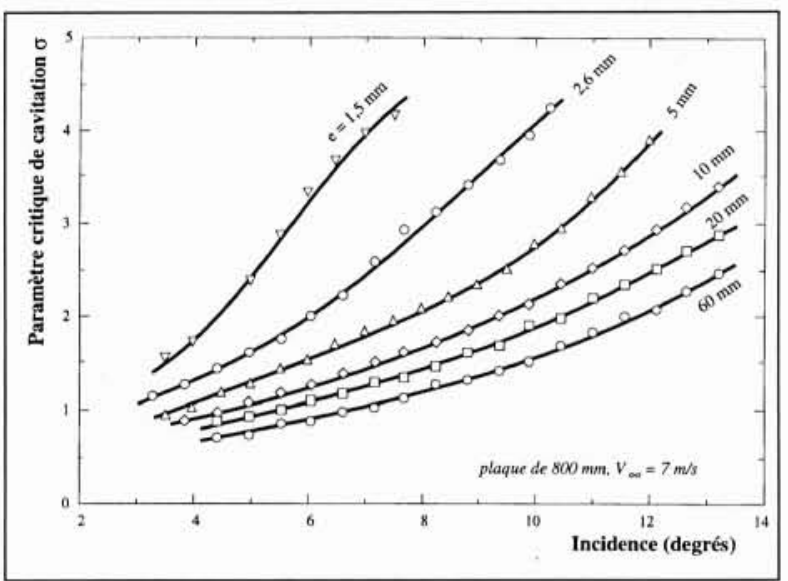

3. Conditions critiques de cavitation en fonction de l'incidence - Effet du confinement.

Aucun résultat n'est présenté pour un jeu d'entrefer inférieur à $1 \mathrm{~mm}$, puisque, dans ce cas, le tourbillon marginal ne cavite plus, comme nous l'avons remarqué au paragraphe 2 .

Les résultats obtenus ici sont très différents des observations réalisées par Gearhart \& Ross [4] ou Farrell \& Billet [5] sur des pompes axiales. Ces auteurs introduisent une valeur réduite $\lambda$, du jeu d'entrefer, calculée comme le rapport entre l'entrefer et l'épaisseur maximale de la section, en bout de pale. Ils mettent en évidence une valeur optimale de $\lambda$ pour laquelle la résistance à la cavitation est la meilleure. Pour des valeurs inférieures, le paramètre de cavitation naissante augmente très sensiblement lorsque l'entrefer diminue, ce qui est la tendance mise en évidence par nos essais. Par contre, lorsque le jeu d'entrefer augmente au-delà de cette valeur optimale, le paramètre de cavitation naissante a également tendance à augmenter, mais de façon plus modérée. Les géométries d'entrefer sur lesquelles ces auteurs travaillent sont cependant très différentes de la nôtre : le paramètre $\lambda$, par exemple, n'est pas défini dans notre cas. La comparaison est donc difficile.

Les observations de Laborde et al. [7] à partir d'essais sur une pompe axiale se rapprocheraient plus de nos résultats, bien que les géométries d'entrefer soient également très différentes. Ces auteurs remarquent que l'élargissement du jeu d'entrefer retarde l'apparition de la cavitation de vortex ; en cavitation plus développée, l'intensité de la cavitation de vortex diminue lorsque la taille du jeu augmente. Le phénomène n'est cependant pas observable dans toutes les configurations.

Remarquons que des mesures de $\sigma$ critique, qui ne sont pas présentées ici, réalisées avec une plaque de confinement beaucoup plus courte à l'amont $(60 \mathrm{~mm}$ en amont du bout d'aile au lieu de $400 \mathrm{~mm}$ ) conduisent à des résultats pratiquement identiques. Ce point confirme le peu d'influence de la couche limite sur la cavitation de tourbillon marginal, du moins dans le domaine de variation des paramètres expérimentaux considéré ici.

42. Evolution de la cavitation en fonction du confinement. Vitesse à l'infini : $7 \mathrm{~m} / \mathrm{s}$ - Nombre de Reynolds : 930000 Incidence : 12 degrés - Paramètres de cavitation : 2,6 


\section{1 PROFILS DE VITESSE TANGENTIELLE}

Parallèlement aux visualisations en régime cavitant, des mesures de vitesse ont été réalisées, en régime subcavitant. L'objectif est de mettre en évidence les modifications que le confinement apporte à la structure subcavitante de l'enroulement tourbillonnaire, d'en estimer, a priori, les conséquences sur le développement de la cavitation et de s'assurer qu'elles sont cohérentes avec les observations réalisées.

Nous avons mesuré les profils de vitesse tangentielle et de vitesse axiale pour diverses conditions d'écoulement. Un soin tout particulier a été apporté à la détermination du centre du tourbillon [6], qui nécessite d'être localisé très précisément pour limiter les sources d'erreur. Les profils de vitesse axiale, généralement difficiles à interpréter, ne sont pas présentés ici. Il n'en ressort pas de conclusions très claires sur la structure de jet ou de sillage du cœur tourbillonnaire, et sur son évolution vers l'aval.

Les profils de vitesse mesurés correspondent à une coupe horizontale, de l'extrados vers l'intrados, c'est-à-dire le long de l'axe y (figure 1), passant par le centre du tourbillon. Il est capital de noter que l'enroulement est loin d'offrir une structure axisymétrique, surtout au voisinage de l'extrémité de l'aile et en situation confinée, comme le montre en particulier la modélisation [3]. Cependant, compte tenu de l'implantation verticale de l'aile et de l'instrumentation disponible, seuls les profils de vitesse suivant l'axe horizontal, y, ont pu être réalisés.

En ce qui concerne la vitesse tangentielle, la figure 4 présente des exemples typiques de profils de vitesse mesurés. On y trouve, à la fois, l'effet du confinement sur les profils de vitesse tangentielle, et leur évolution vers l'aval pour divers jeux d'entrefer. Le graphe correspondant à la station la plus à l'amont, $\mathrm{x} / \mathrm{c}=0,125$, ne comporte pas le profil pour le plus petit entrefer de $4 \mathrm{~mm}$ considéré ici. Cela est dû à une impossibilité de mesure, l'un des faisceaux laser étant coupé par la plaque de confinement.

Les principales tendances mises en évidence par la figure 4 sont les suivants :

- Le confinement induit une augmentation des extrema de vitesse. Son influence est plus sensible côté intrados que côté extrados. Cette augmentation va de pair avec une diminution de pression dans le cœur du tourbillon, comme on peut le prévoir sur la base de l'équation d'Euler, écrite pour un écoulement axisymétrique et projetée sur la direction radiale :

$$
\frac{\partial p}{\partial r}=\rho \frac{V^{2}}{r}
$$

La vitesse apparaissant au carré, toute augmentation de vitesse devrait avoir des répercussions importantes sur les pressions minimales. Cette remarque est cohérente avec les mesures de paramètre critique de cavitation, qui ont montré une très forte influence du confinement sur les conditions de cavitation du tourbillon marginal.

- Le confinement induit une légère augmentation de la taille du cœur visqueux suivant la direction $\mathrm{y}$, parallèle à la plaque. Cela tendrait à indiquer que le confinement provoque une sorte d'aplatissement du tourbillon marginal dans la direction $\mathrm{z}$, perpendiculaire à la plaque, qui s'accompagne probablement d'une certaine expansion dans la direction perpendiculaire, $\mathrm{y}$, où aucune résistance n'est rencontrée.

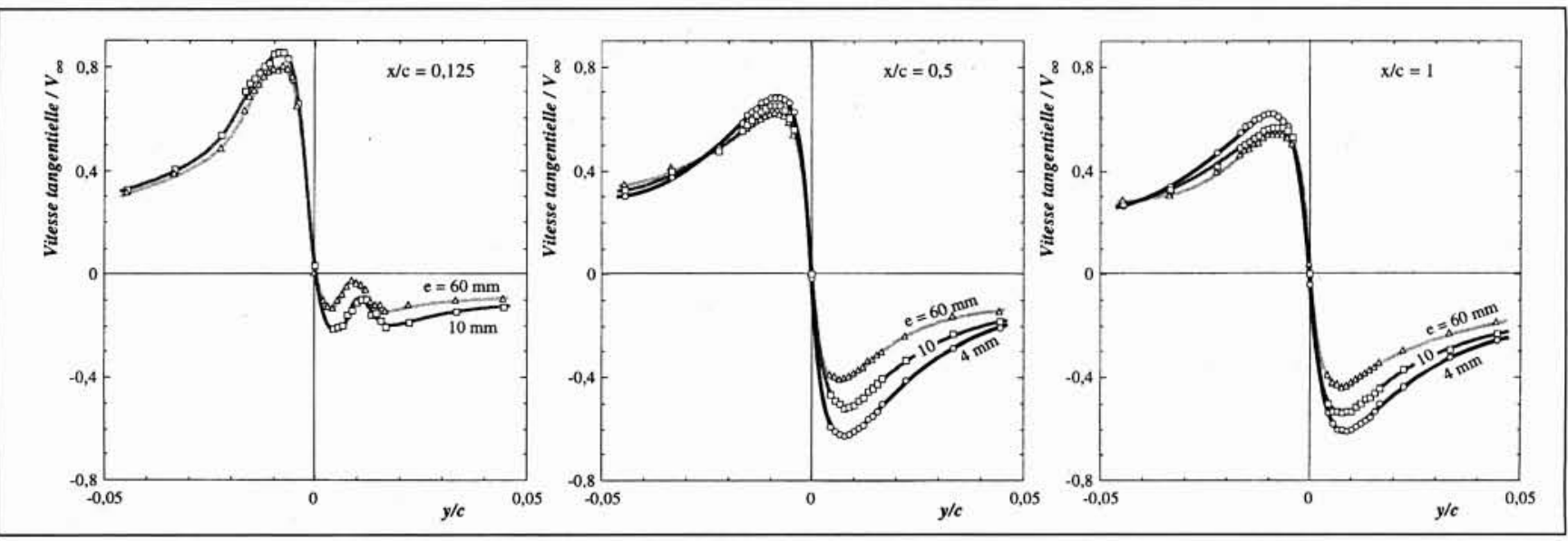

4. Profils de vitesse tangentielle mesurés par anémométrie laser - vitesse à l’infini : $7 \mathrm{~m} / \mathrm{s}$ - Incidence : 8 degrés Influence de la station de mesure vers l'aval et du confinement.

Tableau 1 Extrema de vitesse tangentielle dans le tourbillon pour une vitesse $V_{\infty}$ de $7 \mathrm{~m} / \mathrm{s}$ et une incidence de 8 degrés

\begin{tabular}{|c|c|c|c|c|c|c|c|c|c|c|c|}
\hline $\mathrm{x} / \mathrm{c} \rightarrow$ & 0,083 & \multicolumn{3}{|c|}{0,125} & 0,25 & \multicolumn{3}{c|}{0,5} & \multicolumn{3}{c|}{1} \\
\hline $\begin{array}{c}\mathrm{e} \\
\downarrow\end{array}$ & $\begin{array}{c}\mathrm{C} \\
\left(\mathrm{m}^{2} / \mathrm{s}\right)\end{array}$ & $\frac{\mathrm{V}_{\max }}{\mathrm{V}_{\infty}}$ & $\frac{\mathrm{V}_{\min }}{\mathrm{V}_{\infty}}$ & $\begin{array}{c}\mathrm{C} \\
\left(\mathrm{m}^{2} / \mathrm{s}\right)\end{array}$ & $\begin{array}{c}\mathrm{C} \\
\left(\mathrm{m}^{2} / \mathrm{s}\right)\end{array}$ & $\frac{\mathrm{V}_{\max }}{\mathrm{V}_{\infty}}$ & $\frac{\mathrm{V}_{\min }}{\left(\mathrm{m}_{\infty} / \mathrm{s}\right)}$ & $\begin{array}{c}\mathrm{C} \\
\left(\mathrm{m}^{2} / \mathrm{s}\right)\end{array}$ & $\frac{\mathrm{V}_{\max }}{\mathrm{V}_{\infty}}$ & $\frac{\mathrm{V}_{\min }}{\left(\mathrm{m}_{\infty}^{2} / \mathrm{s}\right)}$ & $\begin{array}{c}\mathrm{C} \\
\left(\mathrm{m}^{2} / \mathrm{s}\right)\end{array}$ \\
\hline $60 \mathrm{~mm}$ & 0,011 & 0,80 & 0,14 & 0,016 & 0,021 & 0,62 & 0,41 & 0,022 & 0,54 & 0,44 & 0,022 \\
\hline $10 \mathrm{~mm}$ & - & 0,85 & 0,22 & 0,020 & 0,022 & 0,65 & 0,52 & 0,025 & 0,57 & 0,54 & 0,024 \\
\hline $4 \mathrm{~mm}$ & - & - & - & - & 0,029 & 0,68 & 0,62 & 0,030 & 0,62 & 0,61 & 0,033 \\
\hline
\end{tabular}


- Les profils de vitesse sont fortement dissymétriques au voisinage du bout d'aile, et tendent à se symétriser vers l'aval. Par ailleurs, le confinement accélère la symétrisation du profil de vitesse. L'accident remarqué sur le côté extrados, pour la station de mesure la plus proche de l'aile, $x / c=0,125$, est probablement dû au sillage. Un calcul purement potentiel de l'enroulement de la nappe en rend compte [3].

$\mathrm{Si}$ l'on se réfêre à un modèle de tourbillon axisymétrique, l'un des paramètres importants est la circulation, $\Gamma$, du tourbillon. Celle-ci définit l'allure du profil de vitesse tangentielle dans la zone potentielle extérieure qui, en milieu infini, est du type :

$$
v=\frac{\Gamma}{2 \pi r}
$$

Si l'on calcule le produit $2 \pi \mathrm{rv}$ en divers points de mesure r, on s'aperçoit généralement qu'il a tendance à tendre asymptotiquement, lorsque $r$ augmente, vers une valeur qui peut être considérée comme caractéristique de la circulation du tourbillon [1]. Cette remarque ne tient qu'en milieu infini. En milieu confiné, on peut montrer [6] que la vitesse décroît plus vite qu'en $1 / r$ et que le raisonnement précédent est difficilement généralisable.

Cependant, pour tenter de caractériser globalement le tourbillon et de lui attribuer une circulation, et plutôt que de se baser sur la partie potentielle extérieure, on peut utiliser les données relatives au cour visqueux. Dans le cas idéal d'un tourbillon de Rankine classique, la circulation est donnée par :

$$
\Gamma=2 \pi a V_{\max }
$$

où a désigne le rayon du cœur visqueux où la vitesse tangentielle atteint son maximum $\mathrm{V}_{\max }$.

Si l'on tente de généraliser cette formule au cas présent, on est amené à introduire une quantité $\mathrm{C}$, homogène à une circulation, et définie par:

$$
\mathrm{C}=\pi \mathrm{D} \frac{\mathrm{V}_{\max }+\left|\mathrm{V}_{\min }\right|}{2}
$$

Dans cette formule, D représente le diamètre du cœur visqueux, assimilé à la distance entre les deux extrema de vitesse. Les quantités $V_{\max }$ et $V_{\min }$ représentent les valeurs des extrema de vitesse. La considération de leur moyenne permet de prendre en compte, dans une certaine mesure, la forte dissymétrie du profil de vitesse. Le tableau 1 indique, pour diverses stations vers l'aval et pour les trois jeux d'entrefer retenus ici, la valeur des extrema de vitesse ainsi que celle de cette circulation $\mathrm{C}$.

Ce tableau appelle deux commentaires :

- Pour un entrefer donné, la circulation $\mathrm{C}$ a tendance à augmenter vers l'aval. Ce fait rend compte du mécanisme d'en-

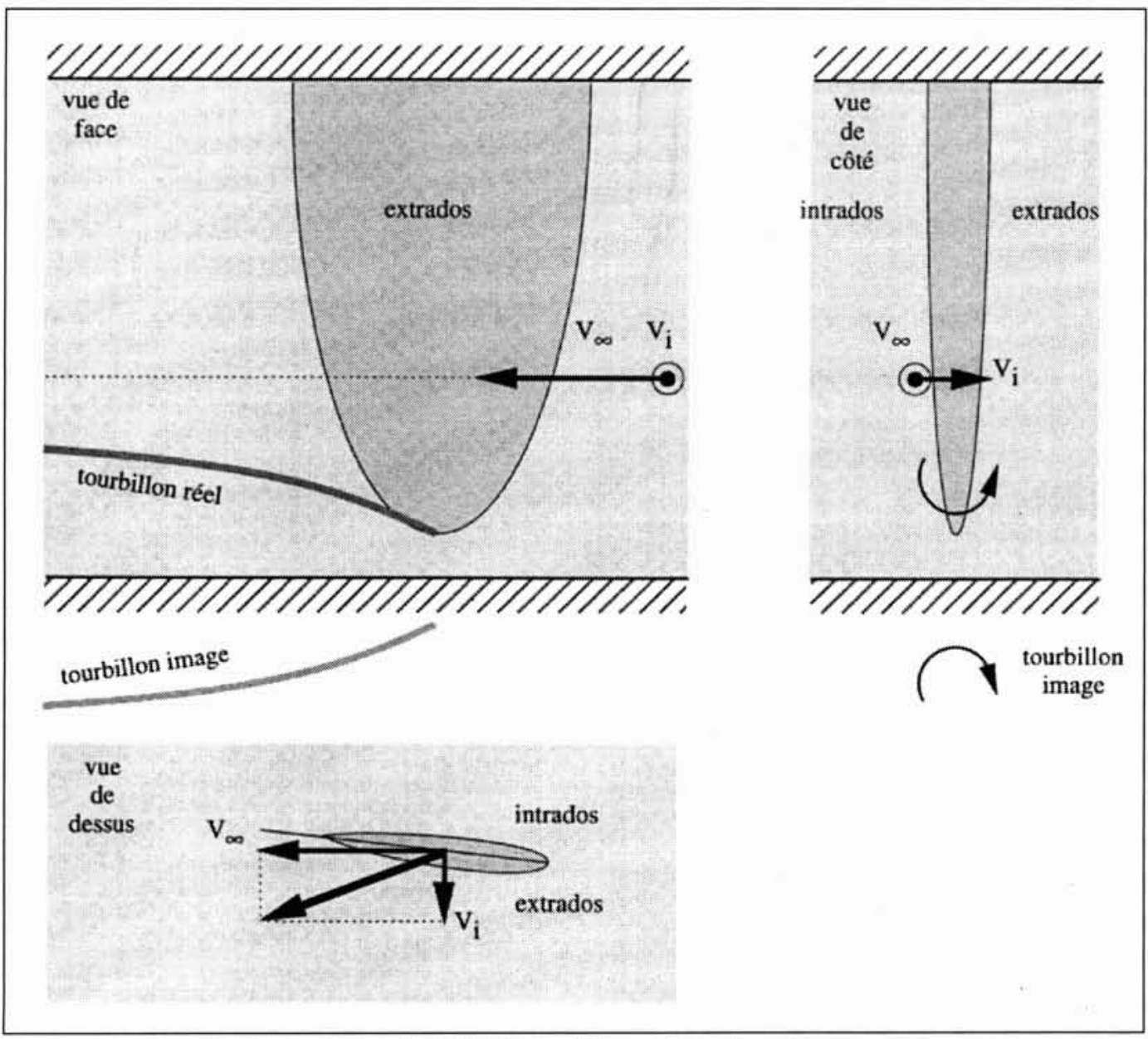

5. Interprétation de l'effet de confinement par la prise en compte du tourbillon image et de la vitesse induite par celui-ci. 
roulement : les filaments tourbillonnaires constituant le sillage de l'aile s'enroulent d'autant plus à l'aval qu'ils sont émis d'un point plus éloigné du bout d'aile, ce qui conduit à une augmentation progressive de la circulation vers l'aval.

- La deuxième observation est plus spécifique du confinement. Pour une station donnée vers l'aval, cette circulation $\mathrm{C}$ augmente avec le confinement. Cette remarque tend à montrer que la diminution du jeu d'entrefer conduit à une augmentation de la circulation du tourbillon. Elle est cohérente avec l'avance à la cavitation observée par confinement.

La grandeur C, telle qu'elle a été définie par l'équation (4), donne donc une mesure globale, qualitativement réaliste, de la circulation du tourbillon. Bien que son intérêt reste assez limité en pratique, elle présente l'avantage d'être applicable à des géométries différentes du milieu infini.

\section{V — DISCUSSION ET INTERPRÉTATION DES RÉSULTATS}

Les résultats précédents peuvent être qualitativement interprétés en considérant l'effet de miroir introduit par la plaque de confinement. Pour que la condition de glissement soit vérifiée sur celle-ci, il faut, d'un point de vue purement potentiel, introduire un tourbillon image fictif, symétrique du tourbillon réel par rapport à la plaque.

Ce tourbillon image induit une vitesse dont la direction est représentée sur la figure 5 . Cette vitesse doit être composée avec la vitesse à l'infini pour connaître la vitesse réelle avec laquelle l'écoulement attaque effectivement l'aile. La vue de dessus montre clairement que la prise en compte de la vitesse induite revient à supposer que l'écoulement attaque l'aile avec une incidence supérieure à l'incidence géométrique. Cet effet est d'autant plus sensible que l'entrefer est plus petit. Par ailleurs, il est d'autant plus petit que la section considérée est plus proche de l'emplanture ; cela revient à supposer que l'aile est, en quelque sorte, "vrillée" par le confinement.

La remarque précédente permet d'expliquer, qualitativement, la majorité des résultats présentés dans ce travail. On comprend d'abord que cette augmentation d'incidence entraîne une augmentation de portance et ainsi une augmentation de la circulation du tourbillon. Cela explique l'évolution observée, en régime non cavitant, des profils de vitesse tangentielle avec le confinement. La conséquence directe sur la cavitation est une avance à l'apparition de la cavitation de tourbillon marginal lorsque l'entrefer diminue.

On comprend également que la cavitation de tourbillon marginal disparaisse dans le cas des très forts confinements. Pour des jeux d'entrefer très petits, l'augmentation d'incidence est si importante que l'écoulement est décollé en bout d'aile, dès le bord d'attaque. Il a une structure de sillage épais en extrémité de pale. Cela explique que la cavitation se manifeste sous la forme d'une cavitation de cisaillement plutôt que d'une cavité attachée. Le bulbe de décollement de bord d'attaque, dont on a pu montrer qu'il jouait un rôle important dans l'initialisation du tourbillon marginal [6], disparaît alors au profit d'un sillage épais dont la nature fortement turbulente conduit probablement à la déstabilisation du tourbillon marginal subcavitant.

\section{VI $\square$ CONCLUSIONS}

Ce travail présente une analyse expérimentale de l'effet du confinement sur la cavitation de tourbillon marginal qui se développe à l'extrémité d'une aile elliptique. Le confinement est dû à une paroi placée à une distance réglable du bout d'aile. Les principaux résultats de l'étude sont les suivants :

- Une campagne de mesure de vitesse par anémométrie laser a montré que les extrema de vitesse tangentielle augmentent lorsque le jeu d'entrefer diminue, et que cette tendance est nettement plus marquée côté intrados. Cette observation tend à prouver que le confinement augmente la circulation du tourbillon marginal.

- Le confinement provoque une nette avance de la naissance de la cavitation dans le tourbillon marginal, par rapport au cas non confiné. Ce résultat est en accord avec la croissance des extrema de vitesse tangentielle observée en milieu confiné, qui prédit une dépression plus forte dans le tourbillon.

- Pour les jeux d'entrefer très réduits, de l'ordre de quelques dixièmes de millimètre, aucune cavitation de tourbillon marginal n'est observée.

- Dans les cas expérimentés ici, aucun effet visqueux lié au développement de la couche limite sur la paroi de confinement n'a pu être mis en évidence. Les effets sont essentiellement de type potentiel : l'ensemble des observations précédentes s'explique qualitativement par la prise en compte d'un tourbillon image qui tend à augmenter l'incidence effective avec laquelle l'écoulement attaque le bout de pale.

\section{RÉFÉRENCES :}

[1] Fruman D.H., Dugué C., Pauchet A., Cerrutti P. \& BriançonMarjollet L., 1992, "Tip Vortex Roll-Up and Cavitation", 19th Symposium on Naval Hydrodynamics, August 24-28, Seoul, Korea.

[2] Deniset F. \& Pellone C., 1996, "Modélisation de l'effet du confinement sur le tourbillon marginal", Troisièmes Journées Cavitation, SHF, 13-14 Novembre 1996, Grenoble.

[3] DeNisET F., 1996, "Modélisation numérique des conditions d'apparition de la cavitation de tourbillon marginal sur une aile-3D - Effet de confinement", Thèse de Doctorat de l'Institut National Polytechnique de Grenoble.

[4] Gearhart W.S. \& Ross J. R., 1991, "Tip leakage effects", Cavitation and Multiphase Flow Forum, ASME FED, 109, 159-164.

[5] FARReLL K.J. \& BIL.ET M.L., 1994, “A correlation of leakage vortex cavitation in axial-flow pumps", Journal of Fluids Engineering, 116, 551-557.

[6] Bouzon O., 1996, "Etude expérimentale de la cavitation de tourbillon marginal : effets instationnaires, de germes et de confinement", Thèse de Doctorat de l'Institut National Polytechnique de Grenoble.

[7] Laborde R., Chantrel. P., Retallleau A., Mory M. \& Boulon O., 1995, "Tip clearance cavitation in an axial flow pump", International Symposium on Cavitation, 2-5 Mai, Deauville, France. 